\title{
Le statut des ministres du culte en France au XIX siècle
}

\section{Brigitte Basdevant-Gaudemet}

\section{OpenEdition}

\section{Journals}

Édition électronique

URL : http://journals.openedition.org/rdr/427

DOI $: 10.4000 /$ rdr.427

ISSN : 2534-7462

\section{Éditeur}

Presses universitaires de Strasbourg

\section{Édition imprimée}

Date de publication : 14 novembre 2019

Pagination : 19-41

ISBN : 979-10-344-0054-6

ISSN : 2493-8637

\section{Référence électronique}

Brigitte Basdevant-Gaudemet, "Le statut des ministres du culte en France au xix ${ }^{\mathrm{e}}$ siècle », Revue du droit des religions [En ligne], 8 | 2019, mis en ligne le 25 novembre 2019, consulté le 19 novembre 2020. URL : http://journals.openedition.org/rdr/427 ; DOI : https://doi.org/10.4000/rdr.427 


\section{LESTATUT DESMINISTRESDUCULTE ENFRANCEAUXIXESIËCLE}

\section{Brigitte BASDEVANT-GAUDEMET}

Université Paris-Saclay, Droit et Sociétés religieuses (DSR)

\section{RÉSUMÉ}

Tout État se préoccupe de la condition des religieux exerçant leurs fonctions sur son territoire. Le souci de contrôle fut particulièrement marqué en France au XIX ${ }^{e}$ siècle où le régime des cultes reconnus permit à la puissance publique de surveiller la formation, la désignation et les comportements de ce personnel religieux. Si l'immense majorité des citoyens adhéraient alors à l'un des quatre cultes reconnus, la surveillance s'étendit aussi aux autres religions ou aux «sectes». L'islam fut, du moins dans les trois départements d'Algérie, organisé selon un système assez comparable à celui des cultes reconnus.

\section{ABstract}

Every state is concerned about the status of religious personnel performing their duty on its territory. The concern for control was particularly marked in France in the 19th century, when the system of recognized religions allowed the public authorities to oversee the training, designation and behaviour of these religious personnel. The vast majority of citizens then adhered to one of the four recognized religions, but surveillance also extended to other religious groups or "sects". In the three departments of Algeria, Islam was organized according to a system quite comparable to that of recognized religions. 
$\mathrm{T}$ raiter des évolutions historiques en France peut sembler hors de propos dans ce volume consacré au droit positif et au culte musulman car cette religion n'était pas, dans le passé, au centre des préoccupations des autorités chargées des cultes. Pourtant, la France comptait de nombreux citoyens musulmans, au Maghreb tout particulièrement. Surtout, préciser ce que connurent les religions historiques constitue un précieux guide pour conduire la réflexion sur les situations actuelles du pluralisme religieux français. Il serait intéressant d'envisager l'époque, passionnante, où l'empereur Constantin reconnut la nouvelle religion ${ }^{l}$ dans l'Empire romain, ouvrant l'ère des faveurs accordées par les empereurs successifs. L'Église chrétienne et les clercs bénéficiaient d'un «statut» fait de privilèges multiples. Ces privilèges, privatae leges, dessinaient les contours d'une condition spécifique pour certains, condition comportant bien des avantages mais pouvant aussi imposer devoirs et interdits. Parallèlement, accordant ce statut aux clercs, l'État romain oblige l'Église à préciser quels sont les clercs et les diverses catégories à l'intérieur du clergé, afin de savoir quelle est la condition civile de chacun ${ }^{2}$. Un statut juridique du clerc se met en place dans l'Empire romain et survit dans l'Occident ${ }^{3}$ chrétien médiéval. Avec des prescriptions perpétuellement revues par le droit canonique, le clergé devient, dès le Haut Moyen-Âge, le premier « ordre» de la Nation. Un groupe particulier, soumis à un droit particulier, qui n'est pas seulement le droit interne de la religion en question, mais qui est aussi constitué par les innombrables dispositions édictées ou simplement admises par le pouvoir royal temporel ainsi que par l'Église et par la société. Le droit des États assigne à tous ces religieux une situation à part. Tel fut «l'ordre»du clergé de notre ancienne France. Aujourd'hui encore, bien des pays reconnaissent aux religieux un statut juridique spécifique. Souvent, un clergé forme une «caste», ou un autre type de communauté, quelle que soit la dénomination. Ce système n'a plus cours en France. Dans la nuit du 4 août 1789, les députés des États généraux ont aboli les droits féodaux, les ordres et les privilèges. La Déclaration des droits de l'homme et du citoyen du 26 août 1789 proclame dans son article 1 que «les hommes naissent et demeurent libres et égaux en droit [...]» et l'article 6 répète que la loi est « [...] la même pour tous, soit qu'elle protège,

1. Édit dit «de Milan», 313.

2. A. JACQUEmin, Le clerc dans la cité: limitation des activités séculières des clercs de Constantin à la fin de l'époque carolingienne, Paris, Cerf, 2016.

3. J. Gaudemet, L'Église dans l'empire romain (IVe-VI ${ }^{e}$ siècles), Paris, Sirey, 1989, p. 152-185 (Histoire du droit et des institutions de l'Église en Occident); pour les périodes ultérieures, $\mathrm{V}$. les autres volumes de cette même collection. 
soit qu'elle punisse $[\ldots] »$. Les congrégations furent expressément supprimées par le décret du 18 août 1792. Inutile de rappeler qu'au cours des années révolutionnaires les clercs ne bénéficièrent d'aucun statut et connurent une situation de clandestinité, d'exil et de persécution.

Ce bref article est essentiellement consacré au XIX siècle, lors du régime des cultes reconnus mis en place par Bonaparte, dans un esprit napoléonien, c'est-à-dire conformément à une politique d'étatisation, de centralisation, d'autorité et de contrôle. Accédant au pouvoir, Bonaparte souhaite la paix sociale; il sait que celle-ci implique de restaurer la paix religieuse et le libre exercice public du culte. Il y procède, selon ses conceptions personnelles, en organisant le «service public» des cultes qui durera jusqu'en 1905. Le concordat du 15 juillet 1801 fut accompagné des Articles organiques, ceux relatifs au culte catholique, mais également ceux concernant les Églises réformées et les Églises de la confession d'Augsbourg. Si les dispositions concordataires étaient parfois demeurées évasives, ce fut en pleine conformité avec la politique de Napoléon qui soulignait qu'une bonne constitution doit être «courte et obscure». Les imprécisions des formules concordataires avaient été corrigées par les Articles organiques. L'ensemble fit l'objet d'une seule et même publication, par la loi du 18 germinal an 10 (8 avril 1802) relative à «l'organisation des cultes». Le culte juif ne sera réglementé qu'en 1808, sur un modèle comparable à celui appliqué aux deux confessions protestantes, mais les rabbins n'émargeront au budget de l'État qu'à partir de 1831. Les cultes reconnus furent donc organisés en institutions publiques, prenant place dans l'ensemble des institutions administratives de l'État, ce qui eut naturellement des conséquences sur le statut des ministres. Telle fut la situation de droit commun des religions au XIX ${ }^{\mathrm{e}}$ siècle. Nous y consacrerons l'essentiel des développements, sans oublier toutefois qu'il y eut, au XIX ${ }^{\mathrm{e}}$ siècle comme par la suite, d'autres religions pratiquées sur le territoire national qui, sans être «reconnues», disposaient de ministres ou de cadres religieux; tel fut notamment le cas de l'islam.

Dans le concordat de 1801 et dans chacune des lois organiques du culte catholique, des cultes protestants et du culte israélite, des dispositions spécifiques sont consacrées aux «ministres». Dès lors, on peut parler d'un statut juridique qui, avec quelques aménagements, persista jusqu'en 1905. Les dispositions concernent la nomination des ministres des cultes (1), mais également les principaux droits ou obligations attachés à la condition de ministre du culte (2). 


\section{NOMINATION DES MINISTRES DU CULTE}

Pour accéder aux fonctions religieuses, un candidat doit satisfaire à certaines conditions (1.1) et être nommé par l'autorité compétente (1.2). Toutes les religions ne sont d'ailleurs pas régies par des principes identiques (1.3).

\subsection{LES CONDITIONS REQUISES DU CANDIDAT}

Le candidat doit répondre à certaines conditions posées par les religions parfois, par les pouvoirs publics également

Dans cette étude, on ne s'attardera pas sur les critères retenus par les religions pour désigner leur personnel. Celles-ci prennent en compte certaines qualités relatives à la vie personnelle: une naissance légitime, une éducation dans la religion concernée, la moralité, éventuellement le célibat, etc. Sous l'Ancien Régime, la doctrine gallicane a comme conséquence de réserver la carrière cléricale aux seuls sujets possédant la nationalité française. La même règle s'impose au XIX ${ }^{e}$ siècle où, par mesure de police, Napoléon exige la nationalité française de tout ministre d'un culte reconnu ${ }^{4}$. Sur ce point comme sur bien d'autres, la loi de 1905 donnera la liberté aux confessions religieuses.

L'une des aptitudes à laquelle les religions et les États se montrent attentifs est la formation du candidat; la question a toujours préoccupé. Au XVI ${ }^{\mathrm{e}}$ siècle, ce fut l'un des objectifs majeurs des Pères au concile de Trente, lorsqu'ils décidèrent la création des séminaires pour les prêtres catholiques ${ }^{5}$. Leur installation effective fut lente. En France, un premier séminaire est ouvert

4. Articles organiques de la Convention du 26 messidor an 9, art. XXXII: «Aucun étranger ne pourra être employé dans les fonctions du ministère ecclésiastique, sans la permission du gouvernement».

5. Concile de Trente, $23^{e}$ session, 15 juill. 1563, can. 18, considéré comme la décision fondamentale de création des séminaires. Le canon contraint chaque évêque à avoir, dans son diocèse, un collège au sein duquel il choisira les enfants qui doivent se destiner aux ordres; l'évêque veillera à leur formation. V. M. VENARD, «Les séminaires en France avant saint Vincent de Paul», in Le catholicisme à l'épreuve dans la France du XVI e siècle, Paris, Cerf, 2000, p. 118-134; V. aussi, B. BASDEVANT-GAudemet, Histoire du droit canonique et des institutions de l'Église latine, $X V^{e}-X X^{e}$ siècle, Paris, Économica, 2014, p. 431-436. Pour la période des cultes reconnus, V. M. LAUNAY, Les séminaires français au XIX ${ }^{e}$ et $X X^{e}$ siècles, Paris, Cerf, 2003; V. aussi, A. Vulllefroy, Traité de l'administration du culte catholique, Paris, 1842, p. 476-487; Répertoire Dalloz, 1853, vº́minaire, retrace précisément les créations successives de séminaires, pour le culte catholique et les Églises protestantes; chacun des volumes de la collection Histoire des diocèses de France (Beauchesne) consacre quelques pages aux séminaires. 
à Reims en 1567, mais le véritable essor de ces établissements diocésains français ne s'effectue qu'à partir du milieu du XvII siècle.

La formation préoccupe aussi l'État qui, parfois, la prend en charge financièrement; l'exemple du régime des cultes reconnus est significatif: pour l'Église catholique, le décret du 23 ventôse an 12 (14 mars 1804) organise les séminaires métropolitains sous la forme «d'écoles spéciales» comme pour la médecine ou le droit. Le rapporteur du décret expose la nécessite de former de «bons citoyens». L'État finance ces séminaires métropolitains; en revanche, il ne dote pas les soixante séminaires diocésains qui sont seulement autorisés. Les directeurs et professeurs des séminaires métropolitains sont nommés par le Premier Consul sur proposition de l'archevêque et des évêques suffragants. Les séminaires diocésains jouissent d'un peu plus d'indépendance à l'égard des pouvoirs publics; ainsi, la nomination des enseignants n'y est pas soumise à validation par le gouvernement. Toutefois, dans tous les séminaires, les responsables doivent envoyer au préfet la liste des inscrits et les professeurs doivent enseigner la Déclaration des quatre articles de 1682, véritable charte du gallicanisme. Les mesures de surveillance policière sont nombreuses. Le décret de 1804 rend obligatoire le passage par les séminaires, du moins pour les principaux dignitaires; il dispose qu'à l'avenir on ne pourra pas être nommé évêque, vicaire général, chanoine ou curé de première classe sans avoir satisfait aux examens dans les diverses matières enseignées dans les établissements métropolitains. Les ministres du culte dont le traitement est prévu dans les textes fondateurs du régime des cultes reconnus sont donc soumis à cette obligation de diplôme qui ne s'impose pas, ou du moins pas dans les mêmes termes, aux ecclésiastiques de rang inférieur.

Ces séminaires métropolitains devinrent faculté de théologie, au sein de l'Université impériale lors de l'organisation de cette dernière par le décret du 17 mars 1808. La désignation des professeurs obéit à une procédure combinant l'intervention de l'Église et de l'État. L'archevêque présente trois candidats et la nomination appartient au Grand Maître de l'Université qui nomme après examen; l'État a le monopole de la collation des grades que sont le baccalauréat, la licence, le doctorat. En 1843, les séminaires français rassemblaient plus de 20000 élèves. Dans les années 1860, on assista à une augmentation sensible des ordinations sacerdotales. Mais, un fort déclin se fit sentir à partir de 1875, déclin suivi d'une crise encore beaucoup plus profonde due à la législation des années 1901, 1904, 1905.

Par la loi du 8 avril 1802 (18 germinal an X) sont également promulgués des Articles organiques relatifs aux cultes protestants; certains portent aussi 
sur la formation ${ }^{6}$ : il est prévu d'instaurer deux séminaires dans l'est de la France pour les luthériens et un autre à Genève pour les calvinistes. Nul ne pourra être nommé pasteur sans y avoir étudié et le gouvernement contrôle naturellement ces établissements qui, dans les faits, se mettent en place et fonctionnent. Le décret du 17 mars 1808 organisant l'Université impériale crée (art. 8) deux facultés de théologie protestante, l'une à Strasbourg pour les Églises de la confession d'Augsbourg et l'autre à Genève pour la religion réformée ${ }^{7}$. Par la suite, divers regroupements et modifications interviendront jusqu'à la réouverture de la faculté de théologie protestante au sein de l'Université à Strasbourg en 1919.

Le décret impérial du 17 mars 1808, qui prescrit des mesures pour l'exécution du règlement du 10 décembre 1806 concernant les Juifs, ne parle pas expressément de la formation des rabbins, mais une école centrale rabbinique sera ouverte à Metz en $1829^{8}$, puis transférée à Paris. Elle est sous l'autorité du consistoire central.

Ce souci de formation n'a rien perdu de son actualité, dans toutes les religions, et notamment l'islam.

6. Articles organiques pour les cultes protestants: titre I: «Dispositions générales pour toutes les communions protestantes», art. 9 à 14 ; la réglementation est précise afin de permettre un contrôle effectif de l'État.

7. Celle-ci sera transférée à Montauban en 1815, lorsque la France perdra Genève.

8. Rapport fait le 4 mars 1905 au nom de la commission relative à la séparation des Églises et de l'État et de la dénonciation du Concordat, chargée d'examiner le projet de loi et les diverses propositions de loi concernant la séparation des Églises et de l'État, par M. Aristide Briand: "Sous Charles X, un arrêté ministériel autorisa, en 1829, l'établissement d'une école centrale rabbinique à Metz. Un règlement fixa le nombre des élèves, le programme des études, le mode d'attribution des diplômes rabbiniques. L'école était placée sous la direction du consistoire de Metz et sous l'autorité du consistoire central de Paris. [...]

Il était pourvu aux frais de premier établissement de l'école sur des fonds réservés du traitement de l'un des plus grands rabbins du consistoire central pour 1827. Les dépenses annuelles étaient payées au moyen d'une allocation au budget du consistoire central, laquelle devait être répartie entre les divers consistoires de province. [...] Outre son rôle administratif, le consistoire départemental a un rôle social; ainsi, il est chargé... par l'arrêté du 17 avril 1832 de surveiller et d'encourager les écoles primaires israélites. [...] Le consistoire de Paris a été chargé par un décret en date du $1^{\text {er }}$ juillet 1859 de l'administration de l'école centrale rabbinique, transférée à Paris le $1^{\text {er }}$ novembre 1859 . Cette école, où sont formés les ministres du culte, est moins un séminaire qu'un établissement d'enseignement supérieur, puisqu'on y est admis qu'à la condition de produire le diplôme de bachelier ès lettres. C'est la faculté de théologie israélite qui délivre des diplômes de licenciés en théologie aux élèves ayant quatre années de scolarité, et, à la fin de leurs études, des certificats d'aptitude au titre de sous-rabbin, rabbin ou grand rabbin (Baugey). Son programme comprend, outre les études religieuses et théologiques, l'histoire de la philosophie, la littérature grecque, la littérature latine, le chaldéen, le syriaque, l'arabe, etc. » 
Nous ne pouvons, dans cet exposé, détailler les qualités requises des personnes qui, sans être «ministre du culte», exercent néanmoins des fonctions pastorales, d'aumônerie, de prédication ou de catéchisme par exemple. Des critères sont également posés, qui dépendent largement de la nature des fonctions exercées, les conditions de candidatures étant plus strictes pour les titulaires de charges pastorales importantes.

La personne répondant à ces conditions doit être nommée par l'autorité compétente, ou parfois, par plusieurs autorités, agissant conjointement.

\subsection{AUTORITÉS PROCÉDANT À LA NOMINATION ${ }^{9}$}

Ne revenons pas à l'inextricable question que fut celle du mode de désignation aux bénéfices ecclésiastiques dans l'ancienne France. Il suffit de dire que si le sujet fut objet de tant de débats souvent conflictuels, c'est en raison de l'importance de son enjeu. Qui est compétent pour nommer ceux qui interviennent auprès des fidèles, en exerçant des fonctions certes religieuses, mais en détenant parallèlement une autorité morale pouvant parfois influer sur les choix politiques du sujet ou du citoyen?

Le système des cultes reconnus établi par Bonaparte attribuait un rôle à chacune des deux puissances, hiérarchie religieuse et pouvoirs publics. Dans cette collaboration des deux pouvoirs, les interventions de l'État ont d'autant plus de poids lorsqu'est en jeu la désignation d'un haut dignitaire. La société française du XIX ${ }^{e}$ siècle était essentiellement catholique. Cette religion possède une hiérarchie stricte, ce qui pour Napoléon présentait entre autres avantages celui de faciliter l'évaluation de l'opportunité des interventions étatiques.

Selon les dispositions du Concordat de 1801, l'évêque est «nommé » par le gouvernement et reçoit l'investiture canonique du pape ${ }^{10}$. Ce système ne pourra résister à la loi de séparation des Églises et de l'État, mais laissera néanmoins des traces. Tant que les cultes sont reconnus, le curé est nommé par l'évêque, mais il doit avoir l'agrément du préfet. Napoléon avait souhaité que les curés soient peu nombreux et avait demandé que soit créée une cure par canton. Pourtant, la France compte, dès l'Empire, de très nombreuses autres églises, notamment à la campagne où il y a pratiquement une église dans chaque village. En droit français, elles n'ont pas le titre de cure, mais

9. F. Messner, P.-H. Prélot, J.-M. Woehrling (dir.), Traité de droit français des religions, Paris, LexisNexis, $2^{e}$ éd. 2013, p. 225-228.

10. Convention conclue entre Sa Sainteté Pie VII et le Gouvernement français; V. les articles 4 et 5 . 
sont des succursales, desservies non par un curé, mais par un desservant. Ce dernier est nommé par l'évêque, qui doit en avertir le préfet ${ }^{11}$. La France comptait environ 31000 desservants à la fin du XIX siècle. À ces responsables de paroisses, il convient d'ajouter les vicaires qui constituent une troisième catégorie de ce clergé paroissial. Nommés par les évêques dans des conditions très similaires à celles des desservants de succursales, ils sont rémunérés par la fabrique mais aussi parfois directement par l'État. Ils dépendent naturellement du curé en titre et, au-delà, de l'évêque.

Dans les Églises protestantes, sans entrer dans les détails, les distinctions entre confessions ou les évolutions de procédure ${ }^{12}$, notons que la désignation des pasteurs fait également intervenir les instances religieuses et les instances étatiques. Des élections, par les conseils presbytéraux ou les consistoires, permettent de dégager des noms de candidats. Ceux-ci doivent être approuvés par le chef de l'État (ou le ministre chargé des cultes au sein du gouvernement). La nomination est subordonnée à certaines conditions fixées par la législation étatique; il s'agit notamment de conditions d'âge, de nationalité française, d'instruction, de certificat de bonne vie et mœurs...

Les critères et modalités de nomination des rabbins sont quelque peu différents. Le grand rabbin est élu par le consistoire central auquel se joignent un certain nombre de dignitaires. Pour les consistoires départementaux, ou encore communaux, le principe électif est aussi essentiel. À ce processus, s'ajoute également une surveillance de la part des pouvoirs publics.

Pour chacun des cultes reconnus, le législateur avait donc posé des règles qui prévoyaient que les autorités publiques étaient appelées à donner leur assentiment. La désignation des hauts dignitaires des confessions religieuses fut soumise à une vigilance sourcilleuse de la part des pouvoirs publics ${ }^{13}$.

11. Rappelons qu'au XIX ${ }^{e}$ siècle la terminologie du droit français ne correspond pas à celle du droit canonique. En droit canonique est considérée comme une paroisse la communauté qui a, à sa tête, un curé. Bonaparte ne voulait pas disperser la fonction curiale à travers de trop petites unités. Contrôler un nombre restreint de curés lui semblait plus aisé. Le curé devait lui-même contrôler les desservants, à la tête des succursales. Le nombre de cures augmenta au cours du régime concordataire, mais la terminologie du droit français demeura inchangée tout au long du siècle. Toutefois, les fidèles et l'opinion publique parlaient couramment de la «paroisse» et du «curé» pour désigner l'église, lieu de culte habituel et le prêtre qui en avait la charge. Ces succursales furent souvent désignées comme étant des paroisses dites de seconde catégorie, confiées non à un «curé», mais à un desservant. Celui-ci dirigeait la paroisse, administrait les sacrements et les fidèles le considéraient comme leur «curé».

12. V. Répertoire Dalloz, 1853, $v^{\circ}$ Culte, $\mathrm{n}^{\circ} 707 \mathrm{~s}$.

13. Ibid., $\mathrm{n}^{\circ} 728$. 
Quelle que soit la religion, ils devaient être français, avoir un réel niveau d'instruction et être choisis en accord entre autorités religieuses et étatiques.

La loi du 9 décembre 1905 met fin au régime des «cultes reconnus», ce qui a comme conséquence immédiate de laisser en principe une entière liberté aux cultes dans le choix de leurs ministres, dès lors qu'il n'y a pas atteinte à l'ordre public défini par le législateur. Cette liberté de nomination est-elle totale? Je rappelle seulement deux exceptions en ce qui concerne les désignations épiscopales: d'une part, le chef de l'État a conservé la prérogative consistant à «nommer» l'archevêque de Strasbourg et l'évêque de Metz, dans les deux diocèses qui revinrent à la France en 1918. D'autre part, pour les autres diocèses de l'Hexagone, le gouvernement bénéficie d'un «droit de regard»; de fait, selon la procédure mise en place par le modus vivendi du 20 mai 1921, le nonce demande au gouvernement français «s'il n'a pas d'objection à faire valoir d'un point de vue politique à l'égard du candidat pressenti par le Saint-Siège». Dans la première moitié du $\mathrm{xx}^{\mathrm{e}}$ siècle, des réserves ont pu être présentées et Rome en a tenu compte. Depuis Vatican II on imagine mal qu'un gouvernement recourt à cette procédure et l'on peut dire - qu'en fait sinon en droit - le pape nomme librement les évêques en France ${ }^{14}$. Il ne revient pas à l'historienne d'évoquer davantage le $\mathrm{xx}^{\mathrm{e}}$ siècle.

\subsection{LES CULTES NON-RECONNUS}

$\mathrm{Au} \mathrm{XIX}^{\mathrm{e}}$ siècle, certains mouvements ou religions n'ont pas accès au régime des «cultes reconnus» et sont expressément qualifiés par l'Administration de «cultes non-reconnus». Il s'agit souvent de religions venant de l'étranger ou de groupes considérés comme des «sectes», ou encore de l'islam.

Anglicanisme, orthodoxie, presbytérianisme écossais et d'autres minorités encore sont présents sur le sol français et plusieurs sont considérés comme des «sectes» ${ }^{15}$. Le personnel de ces mouvements, qu'ils soient qualifiés de religions ou de sectes, doit se couler dans le moule du droit commun.

14. R. Metz, «Le Président de la République française, dernier et unique chef d’État au monde qui nomme les évêques», Revue des sciences religieuses, 60/1-2, 1986, p. 63-89.

15. P.-O. Chaumet, «L'administration des cultes "non-reconnus" par l'État au XiX siècle (1802-1905)», Revue historique de droit français et étranger, 2006 (1), p. 19-45: l'auteur évoque de façon très pertinente bon nombre d'affaires aboutissant à un accord - ou à un refus - de la part des pouvoirs publics; F. ZARLENGA, Le régime juridique des cultes non reconnus dans la France concordataire (1801-1905), thèse, Paris-Est-Créteil, mars 2018. 
Ce droit commun s'applique notamment aux réunions qui peuvent être organisées. Le Code pénal de 1810 autorise les réunions religieuses de moins de vingt personnes, mais celles qui rassembleraient un plus grand nombre de participants devaient recevoir l'agrément du gouvernement et celui du maire, sous peine de poursuites pénales ${ }^{16}$. Sous le Second Empire, le décret impérial du 19 mars 1859 accorda un peu plus de liberté, en prévoyant que les autorisations pouvaient être concédées de façon perpétuelle et non plus pour chaque réunion. Avant comme après le décret de 1859, les autorisations de réunions furent souvent accordées, mais les refus furent eux aussi nombreux. Ils étaient officiellement motivés par la crainte d'atteinte à la tranquillité publique et résultaient parfois, même si cela ne fut jamais dit officiellement, d'une suspicion de mise en cause politique du gouvernement. La liberté religieuse demeure donc très «encadrée » pour les ministres de ces cultes étrangers, non-reconnus. L'assouplissement de la réglementation prévu en 1859 bénéficia davantage aux demandes émanant de responsables religieux français, les étrangers demeurant soumis à plus de surveillance. Les ministres de ces cultes étant souvent étrangers, ils firent donc, au XIX ${ }^{e}$ siècle, l'objet d'une surveillance policière constante, plus ou moins stricte selon les périodes. En outre, ces étrangers devaient au préalable obtenir l'autorisation de séjourner en France; celle-ci fut généralement accordée, mais les refus ne furent pourtant pas exceptionnels.

Dans l'histoire, l'organisation de l'islam et le statut de ses ministres n'a guère fait l'objet de débat pour ce qui concerne le territoire métropolitain de la France. Toutefois, la question se posa dans d'autres territoires soumis à la France, notamment dans les trois départements d'Algérie, après la conquête de 1830. Rapidement, les gouvernements français manifestèrent un fort désir de contrôle, d'autant qu'ils voyaient parfois dans l'islam un obstacle à la domination de la France ${ }^{17}$. L'islam est salué à la Chambre des députés dès 1931, mais sa gestion reste dans la sphère des affaires coloniales, c'est-à-dire avec des règles dérogatoires au droit commun; parallèlement, le Répertoire Dalloz en fait état à la rubrique «Association illicite». Pendant une vingtaine d'années, la situation demeura assez confuse. Puis, avant même le coup d'État du 2 décembre 1851, le gouvernement, déjà sous l'autorité

16. Art. 291 du Code pénal de 1810. L'article 294 interdit également ces réunions dans des maisons ou appartements privés.

17. O. SAAÏDIA, «L'invention du culte musulman dans l'Algérie coloniale au XIX siècle», L'Année du Maghreb, 2016, p. 115-132; F. FREGOSI, «Islam et État en Algérie. Du gallicanisme au fondamentalisme d'État», Revue du monde musulman et de la Méditerranée, $\mathrm{n}^{\circ} 65$, L'Algérie incertaine, 1992, p. 61-76; B. ÉTIENnE, La France et l'Islam, Paris, Hachette, 1989. 
du Prince Président futur Napoléon III, publia une circulaire en date du 17 mai 1851 dont l'objet était l'organisation du culte musulman: celui-ci devait se dérouler dans des mosquées répertoriées et sous la conduite d'un personnel adéquat. La circulaire demandait qu'existe un personnel qualifié constitué de personnalités supérieures, à côté d'autres personnes, qui restaient inférieures; c'était tenter de faire naître une sorte de hiérarchie, comparable au modèle catholique et, naturellement, plus facile à contrôler. Le même texte prévoyait que des desservants soient nommés par l'État, rétribués par lui. Des indemnités furent effectivement instaurées et versées. En somme, il s'agissait d'une sorte d'extension du modèle concordataire. Le schéma était fort mal adapté et impliquait, en outre, l'existence d'interlocuteurs officiels. De 1860 à 1870, Napoléon III entretint des liens étroits avec Abd el-Kader, ce qui lui permit de s'immiscer fréquemment dans la vie de ces trois départements. En 1870 fut instaurée une "commission d'administration et de surveillance des mosquées de la ville du département d'Alger»; une autre commission fut mise en place pour la ville de Constantine ainsi qu'à Oran. Pourtant, dans cette dernière ville, les structures demeurèrent bien imprécises. Ces commissions s'occupaient essentiellement des questions matérielles et n'envisageaient guère la désignation, la rémunération, ou les fonctions des ministres du culte. S'il reste délicat de cerner précisément l'organisation de l'islam dans les départements d'Algérie, constatons néanmoins l'intérêt que les gouvernements français portèrent à la situation et leur volonté d'exercer pour le moins un droit de regard sur la désignation des imams et autres personnalités, en accordant, parallèlement, un certain financement à ce personnel religieux musulman sur budget public. En 1900 encore, alors que l'anticléricalisme était à son apogée dans l'Hexagone, les établissements musulmans de diverses natures sont florissants en Algérie, voulus et dotés par le gouvernement français qui nomme et contrôle ces religieux formés dans les trois médersas alors existantes ${ }^{18}$. Dans ces médersas, il s'agit de former des religieux capables de guider leurs coreligionnaires, mais qui soient en même temps des personnalités pouvant rendre à l'autorité française les services qu'elle attend d'elles. Aucune loi n'intervint pour étendre expressément le régime des cultes reconnus à l'organisation de l'islam en Algérie; toutefois, par ces diverses mesures, la situation du culte musulman dans ces

18. E. DoutTé, L'Islam algérien en l'an 1900, Paris, Hachette, 1900, p. 117-132. L'auteur énumère le personnel affecté à chacune des mosquées dans chacun des trois départements et décrit les établissements de formation de ces fonctionnaires du culte musulman. Les trois médersas sont à Alger, Tlemcen et Constantine. Elles rassemblent des professeurs français et d'autres musulmans. 
départements fut pratiquement identique à celle que connurent les cultes officiellement reconnus sur le territoire métropolitain.

Dans les trois départements algériens, la loi de 1905 ne mit pas fin à cette tutelle étatique. Le décret du 27 septembre 1907 prévoyait l'extension de la loi de 1905, mais la réalité fut autre. Le culte musulman resta sous contrôle du gouvernement français. La circulaire du 16 février 1933 continuait à consentir des indemnités au personnel cultuel, en contrepartie de quoi l'État accordait des agréments aux prêches prononcés dans les mosquées domaniales. À plusieurs reprises, les musulmans demandèrent à bénéficier de la loi de 1905 et de l'indépendance qui en est la conséquence. Telle fut notamment la requête de l'association des oulémas réformistes du cheikh Ben Badis dans les années 1920-1930. Pourtant, le gouvernement ne céda pas et le contrôle persista ${ }^{19}$. La loi du 20 septembre 1947, portant statut organique de l'Algérie, réaffirmait l'indépendance du culte musulman à l'égard de l'État dans le cadre de la loi de 1905. Cette déclaration de principe n'eut pas plus de conséquences car l'État entendait maintenir son contrôle. Il y tenait d'autant plus qu'il refusait la citoyenneté aux musulmans; il s'ensuivait nécessairement que les ministres du culte musulman devaient assurer un certain rôle d'agent public, par exemple pour la gestion de l'état civil. Cette attitude du gouvernement français entrait dans le cadre de sa politique coloniale plus qu'elle ne résultait de considération religieuse. Antoine Sfeir et René Andrau estiment que cette non-séparation de la religion et de l'État en Algérie eut une certaine influence par la suite dans le maintien des liens étroits entre religion et société civile que l'on retrouve aujourd'hui. Ajoutons qu'elle correspondait aussi, en un sens, à la tradition algérienne de lien entre État et religion, ceci avant la conquête française. Du reste, ce lien sera rétabli après l'indépendance dans la Constitution algérienne de 1963 qui consacrera l'islam comme religion d'État. Parallèlement, le régime des indemnités versées aux imams se maintiendra après l'indépendance, mais le nombre des bénéficiaires diminuera considérablement et les indemnités seront d'un faible montant.

Plus largement, en 1955-56, le développement d'aumôneries militaires musulmanes avait été envisagé ${ }^{20}$. Les textes en vigueur le permettaient pour les colonies ou autres territoires français. D’autre part, des imams étaient déjà

19. A. Sfeir et R. Andrau, Liberté, Égalité, Islam. La République face au communautarisme, Paris, Tallandier, 2005, spéc. p. 35-36.

20. X. Boniface, L'aumônerie militaire française, Paris, Cerf, 2001; sur les propositions sans suite pour créer une aumônerie militaire musulmane en Algérie, V. p. 472-474. 
présents dans les armées égyptienne, irakienne et jordanienne. Pourtant des réticences se faisaient sentir. Ceux qui estimaient la création inopportune invoquaient l'absence de clergé et de hiérarchie dans l'islam et donc la difficulté des modalités de désignation. À cela s'ajoutait parfois, notamment pour l'Algérie, la crainte que cette aumônerie n'entretienne l'hostilité à l'égard de la France. Pour l'Algérie, ces aumôneries militaires musulmanes ne furent pas mises en œuvre.

Si les pouvoirs publics intervenaient fortement dans le choix des ministres du culte, dans leur formation puis leur désignation, ces mécanismes eurent naturellement des conséquences sur les droits et obligations de ce personnel religieux. L'État napoléonien s'était préoccupé de doter la puissance publique de procédures de contrôle permettant d'intégrer ce personnel religieux aux «agents de l'État». Ces mécanismes, qu'ils soient avantages ou contraintes à l'égard des intéressés, subsistèrent tout au long du Xix $x^{\mathrm{e}}$ siècle.

\section{OBLIGATIONS, DROITS ET SANCTIONS SPÉCIFIQUES CONCERNANT LES MINISTRES DU CULTE}

\subsection{LES OBLIGATIONS}

Dans le régime concordataire, les ministres des cultes reconnus sont soumis à certaines obligations à l'égard de l'État, devoirs assez peu nombreux, même s'ils furent susceptibles de présenter de véritables contraintes.

Tout ministre d'un culte est tenu par une obligation de fidélité à l'État. Le régime concordataire imposait de prêter serment devant le chef de gouvernement ou devant le préfet ${ }^{21}$, et de s'engager à dénoncer tout complot contre l'État. L'Église était bien le service public voulu par l'empereur et ses prêtres devaient participer à l'œuvre administrative d'ensemble afin d'assurer ordre, cohésion et hiérarchie dans l'État comme dans la société. En pratique, les curés ne prêtèrent pas le serment prévu. Les évêques ne le prêtèrent que dans les premiers temps. Quant aux missions de police que Napoléon

21. Art. 6 du Concordat: «Je jure et promets à Dieu, sur les saints évangiles, de garder obéissance et fidélité au Gouvernement établi par la Constitution de la République française. Je promets aussi de n'avoir aucune intelligence, de n'assister à aucun conseil, de n'entretenir aucune ligue, soit au dedans, soit au dehors, qui soit contraire à la tranquillité publique; et si, dans mon diocèse ou ailleurs, j'apprends qu'il se trame quelque chose au préjudice de l'État, je le ferai savoir au Gouvernement. » Le serment devait être prêté par les évêques, mais aussi par les ecclésiastiques du second ordre. 
attendait du clergé, elles ne furent jamais remplies. Cependant, les ministres du culte ne pouvaient être ni jurés ni avocats et ne pouvaient pas davantage exercer des fonctions judiciaires.

On sait que les Articles organiques avaient comme objectif premier, et presque unique, de soumettre l'exercice du culte au contrôle des pouvoirs publics. En conséquence, ils énuméraient un certain nombre d'obligations auxquelles le clergé devait se plier.

L'obligation de résidence se révéla souvent la plus contraignante. Au $\mathrm{XIX}^{\mathrm{e}}$ siècle, elle s'imposait aux évêques qui ne pouvaient pas sortir de leur diocèse sans autorisation du gouvernement, ainsi qu'aux curés contraints de résider dans leur paroisse ${ }^{22}$. Cette règle avait existé sous l'Ancien Régime, afin de contraindre le pasteur à s'occuper de son troupeau, dans son diocèse, mais la prescription n'était guère respectée. Au XIx siècle, ce sont des préoccupations de police qui dictent la règle: il s'agit d'interdire à l'épiscopat de se réunir pour éviter qu'il ne prenne des positions peu conformes aux vues politiques des gouvernements. En pratique, la prohibition constitua un frein sévère à tout concile provincial. En France, des conciles provinciaux se tinrent seulement au cours des années 1849 à 1851. S'ils furent alors autorisés, ce fut en raison du mouvement éphémère d'idylle entre Église et État qui ne constitua qu'une parenthèse de courte durée dans l'ensemble du siècle. L'interdiction de s'assembler englobait toute réunion de concertation entre évêques. Il arriva même que les gouvernements étendent cette prohibition de réunion à la correspondance, surveillant et sanctionnant donc les lettres que les évêques pouvaient s'adresser les uns aux autres.

L'article 4 des Articles organiques visait expressément l'interdiction de tous conciles, synodes et autres assemblées délibérantes qui ne pouvaient se tenir sans autorisation du gouvernement.

Dès décembre 1905, la liberté de réunion retrouva son domaine d'application.

\subsection{LES DROITS}

Contrôlés, mais également protégés, les ministres des cultes reconnus jouissent de certains droits ou avantages dont nous n'évoquons que les principaux.

22. Articles organiques, art. 20 pour les évêques et art. 30 pour les curés. 
Le traitement apparut souvent comme l'une des principales faveurs ${ }^{23}$. Les ministres des cultes reconnus sont rémunérés par l'État. Par l'article 14 du Concordat, l'État s'engageait à assurer un «traitement convenable» aux évêques et aux curés ${ }^{24}$. Le traitement des desservants et vicaires n'était pas envisagé par la convention signée avec le Saint-Siège, mais il le fut par les Articles organiques et par le décret du 11 prairial an XII. Il fut toujours faible, s'élevant à environ la moitié de celui des «curés» en titre. Ces cures étaient d'ailleurs réparties entre deux classes distinctes ayant chacune leur taux de rémunération. Les Articles organiques des cultes protestants prévoyaient le traitement des pasteurs. Quant aux rabbins, ils ne furent rémunérés qu'à partir de la Monarchie de Juillet ${ }^{25}$. Ces salaires fluctuèrent, au gré des politiques plus ou moins cléricales ou anticléricales des gouvernements. Ils furent supprimés en 1905, suppression considérée comme une victoire de la part des républicains et qui plongea bien des clercs dans un profond désarroi.

La question de la nature juridique de cette rémunération ne fut jamais clairement tranchée. Les débats furent parfois animés et les répercussions importantes. S'agit-il d'un traitement, versé en raison d'un service fait? Le caractère de «service public» des cultes reconnus constitue un argument de poids à l'appui de cette thèse. Toutefois, l'Église catholique soutient pour sa part que ces rémunérations ne sont que la compensation de la confiscation des biens de l'Église prononcée lors de la Révolution; la Constitution civile du clergé (12 juillet 1790) avait en effet prévu ces versements à la suite du décret du 2 novembre 1789 nationalisant les biens du clergé.

L'enjeu des discussions importe, mais celles-ci n'aboutirent jamais à une solution acceptée par tous. Rémunéré par l'État, le ministre du culte était-il un «fonctionnaire»? C'est alors la qualification juridique de son statut qui est en débat. Si la rémunération n'est que la compensation des confiscations, elle ne peut être assimilée à un salaire de fonctionnaire. Mais, même dans l'hypothèse où l'on admettrait que les sommes dues soient un véritable traitement, est-ce celui d'un «fonctionnaire»? La question était d'autant plus délicate, qu'il n'y avait pas, au XIX ${ }^{\mathrm{e}}$ siècle, de définition, et encore moins de

23. F. Messner, Le financement des Églises: le système des cultes reconnus (1801-1983), Strasbourg, Cerdic, 1984. L'auteur analyse précisément chacun des cultes reconnus, les évolutions dans le temps et les différences selon les fonctions exercées dans le culte en question.

24. Sous le Premier Empire, il y eut environ 3500 à 4000 curés, alors que le royaume en comptait environ 37000 à la fin de l'Ancien Régime.

25. Loi du 8 février 1831 qui organise la rémunération du plus grand nombre de rabbins mais pas de tous. 
statut, du «fonctionnaire ${ }^{26}$. Pourtant, les pouvoirs publics et les autorités politiques considéraient que qualifier le ministre du culte de «fonctionnaire» permettait de renforcer la surveillance à son égard. De son côté, l'Église n'admit jamais cette thèse, considérant que le lien de subordination liant un ministre du culte à sa hiérarchie ou plus largement à sa religion était incompatible avec la qualité de fonctionnaire, conception que l'on retrouve aujourd'hui.

En tout état de cause, l'un des objectifs essentiels de la politique anticléricale des républicains était la suppression du budget des cultes. De fait, toute rémunération cessa à partir de la loi de 1905, du moins là où la séparation s'appliqua. Actuellement, le droit local des cultes alsacien-mosellan maintient une rémunération aux ministres des confessions catholique, protestantes et juive.

Une fois nommé, le ministre du culte reconnu bénéficie-t-il d'une stabilité, lui permettant d'exercer sa charge avec une relative indépendance vis-à-vis d'une autorité religieuse et vis-à-vis des pouvoirs publics? Le juriste du $\mathrm{XxI}^{\mathrm{e}}$ siècle pourrait penser que la réponse à la question était commandée par la qualité de fonctionnaire, attribuée - ou non attribuée - au personnel religieux. Telle ne fut pas exactement la situation, ne serait-ce qu'en raison de l'apparition tardive de la règle d'inamovibilité des fonctionnaires. Les statuts furent divers.

Le curé d'une paroisse catholique était inamovible ${ }^{27}$; il jouissait d'une stabilité que le clergé de l'ancienne France avait ardemment demandée, mais sans jamais l'obtenir. Sa destitution, sanction grave, impliquait la mise en œuvre d'une procédure lourde, très peu usitée au cours du XIXe siècle. La nomination nécessitant l'accord des autorités ecclésiastiques et séculières, la destitution devait suivre la même procédure et se faire avec l'accord des deux pouvoirs. Ce lourd mécanisme ne fut qu'exceptionnellement mis en œuvre.

Desservants ou vicaires pouvaient être librement déplacés ou révoqués par l'évêque. Au cours du siècle, ces mutations furent fréquentes; elles se déroulèrent parfois dans un climat d'entente cordiale; elles furent plus souvent perçues par le prêtre déplacé comme une sanction et le clergé du second

26. B. Martin-Gay, Le coup d'État en permanence? L'agent public et l'enjeu césarien de la candidature officielle sous le Second Empire, Paris, Boccard, 2015, spéc. p. 41 à 100.

27. C'est du moins ce qu'il faut entendre par le silence du Concordat et des Articles organiques. De fait, l'article 31 des organiques prévoit expressément cette possibilité de révocation pour les autres prêtres séculiers. 
ordre dénonçait fréquemment l'autoritarisme épiscopal de l'époque. Jamais les pouvoirs publics n'osèrent critiquer l'autorité diocésaine sur ce point.

Le pasteur protestant jouit d'une certaine stabilité. Une fois élu, il ne peut être destitué que si le conseil presbytéral qui l'a choisi présente des motifs légitimes de destitution, motifs soumis à l'appréciation du gouvernement qui les accepte ou les refuse.

Le grand rabbin du consistoire central bénéficie d'une totale stabilité puisqu'il est élu à vie.

L'objet de ce court article n'est pas de détailler les procédures de destitution, révocation, déplacement des uns ou des autres. Celles-ci varièrent selon les époques, selon les religions et selon la fonction du ministre du culte ou sa place dans une éventuelle hiérarchie. Remarquons seulement que l'État ne disposait en ce domaine que de prérogatives réduites consistant, généralement, à ratifier la décision d'une autorité religieuse. Les pouvoirs publics n'avaient pas - ou n'avaient qu'exceptionnellement - l'initiative de la mise en œuvre de tels processus. Bien qu'il accordât un traitement à ceux qu'il considérait comme chargés d'un service public, l'État n'avait pas toute liberté pour les déplacer ou les destituer. Nous verrons qu'il possédait d'autres moyens pour assurer son autorité.

Curé, prêtre, desservant, pasteur, rabbin, tout ministre du culte dispose d'un large «droit de police» sur le lieu de culte qu'il gère et dont il est, dès le XIX ${ }^{e}$ siècle, l'affectataire. À l'intérieur de l'édifice, son droit de police est assez étendu, dès lors qu'il l'exerce en accord avec son supérieur hiérarchique dans le cadre de la confession en question. Des limites à sa liberté de décision découlent évidemment des risques d'atteinte à la sécurité ou à l'ordre public. Il convient néanmoins d'ajouter que les Articles organiques contiennent de nombreuses mesures pointilleuses sur la liturgie, le catéchisme, les prières publiques, les places réservées dans les églises, les sonneries de cloches, etc. Tout discours politique y est également interdit. Dans le régime concordataire, l'usage des bâtiments et le déroulement des cérémonies religieuses, même à l'intérieur de l'édifice, ne relèvent donc pas uniquement de la libre décision du curé ou du desservant. Aux termes de l'article 45 des Articles organiques, le ministre du culte ne peut pas non plus organiser de manifestation religieuse, c'est-à-dire de procession, à l'extérieur des bâtiments dans les villes possédant des temples affectés à diverses religions. La disposition ne sera pas toujours strictement appliquée, mais elle permettra pourtant aux maires ou préfets anticléricaux de demander la répression éventuelle de sa violation. 
La loi municipale de 1884 précise divers points relatifs à la police des cultes, dans un esprit anticlérical conforme à la politique des républicains alors au pouvoir. Ainsi, au XIX $x^{e}$ siècle, pour ne citer qu'un seul sujet souvent source de conflits vers la fin du siècle, il était interdit à un curé ou un desservant curé de critiquer la politique scolaire des républicains. De telles limites à la liberté de parole disparurent au $\mathrm{xx}^{\mathrm{e}}$ siècle.

En effet, loi de liberté, la loi de 1905 accordera au ministre du culte affectataire un pouvoir de police beaucoup plus étendu; sa liberté d'action et de parole ne connaît que quelques rares limites. L'article 26 de la loi de 1905 dispose qu'il «est interdit de tenir des réunions politiques dans les locaux servant habituellement à l'exercice d'un culte», mais cette disposition n'est assortie d'aucune sanction alors qu'au siècle précédent les pouvoirs publics pouvaient considérer qu'il y avait atteinte portée à l'ordre public dès que le desservant professait quelques paroles critiques à l'égard du pouvoir politique. À partir de 1905, cette preuve de l'atteinte à l'ordre public fut plus difficile à apporter et les célébrants jouirent d'une plus grande liberté d'expression puisqu'aucune sanction n'était prévue en cas de violation des dispositions de cet article 26.

Ce droit de police du culte appartient à «l'affectataire» du bâtiment. La question peut se poser de savoir quel est cet «affectataire», notamment lorsque plusieurs personnes revendiquent ce titre, hypothèse qui ne fut pas exceptionnelle. Dans le régime des cultes reconnus, les modalités de désignation étant assez précisément définies, il suffisait généralement de vérifier la régularité de la nomination. À partir de 1905, l'État ne «reconnaissant » aucun culte, il devenait impossible de «reconnaître» un «ministre du culte». Pourtant, la subtilité du droit français et plus particulièrement des jurisprudences tant de la Cour de cassation que du Conseil d'État régla rapidement divers points ${ }^{28}$. Il arriva que deux prêtres s'opposent, pour savoir lequel avait autorité sur une église, pouvait y organiser le culte et avait, parallèlement, la jouissance du presbytère. Le Conseil d'État trancha sans ambiguité: le ministre du culte légitimement affectataire est celui qui est considéré comme tel par la hiérarchie. La Haute Assemblée s'interdit à elle-même d'intervenir dans des questions relevant de l'organisation interne du culte et se référa systématiquement à la décision de l'autorité religieuse hiérarchique. Au lendemain de la loi de Séparation, de tels conflits surgirent au sein de l'Église catholique où cette hiérarchie est clairement établie. Le juge s'en remit systématiquement à l'opinion de l'évêque. Ainsi, l'autorité publique peut prendre

28. F. Messner, P.-H. Prélot, J.-M. Woehrling (dir.), op. cit., p. 253. 
en compte la qualité de ministre du culte, ou de religieux, même si le droit français ne définit pas ces concepts; mais ce n'est pas elle qui détermine les éléments constitutifs de cette qualification. Le droit français la prend comme un fait, dépendant de la seule autorité religieuse.

Un exemple d'un droit particulier dont peuvent bénéficier les ministres du culte est constitué par l'exemption du service militaire. La loi Jourdan de 1798 avait instauré l'obligation du service militaire universel, selon le système de la conscription. Dès 1802 fut instauré un tirage au sort avec possibilité de «remplacement». La durée du service variait selon les périodes et fut souvent de trois ans.

Les Articles organiques de chacune des confessions prévoyaient expressément l'exemption des ministres des cultes et des séminaristes. Parmi les lois de laïcisation des républicains de la fin du siècle, en 1889, une loi réduisit ce privilège; la durée normale du service militaire était alors de trois ans: les ministres du culte ne furent plus totalement exemptés, mais leur obligation fut limitée à une année.

Par la suite, la France ne s'est jamais montrée très favorable à l'objection de conscience; pourtant celle-ci a été reconnue et son statut a été organisé par la loi du 21 décembre $1963^{29}$. Ajoutons que la Commission européenne des droits de l'homme ${ }^{30}$ a estimé que les États avaient le droit de ne pas admettre que les objecteurs de conscience ne fassent aucun service militaire, dès lors que celui-ci pouvait être remplacé par un service civil.

Un autre sujet fondamental fait l'objet d'une contribution spécifique dans ce volume à laquelle il suffit de renvoyer : la condition particulière du ministre du culte face au droit du travail ${ }^{31}$.

Tels sont quelques-uns des principaux privilèges des ministres du culte, dans une liste qui n'est certes pas exhaustive. Tenus à certaines obligations, possédant certains droits, ceux-ci encourent aussi certaines sanctions spécifiques.

29. J. Duffar, «L'objection de conscience en droit français», in Conscientious Objection in the EC Countries, Milano, Giuffrè, 1992, p. 45-89.

30. Commission supprimée en 1998 lorsque la Cour devint permanente.

31. V. dans ce volume, Ph. Auvergnon, « Ministres du culte et exclusion du contrat de travail: à propos d'un changement de paradigme», p. 93. 


\subsection{DES SANCTIONS SPÉCIFIQUES}

Mentionnons trois exemples de sanctions, parmi les plus importantes et de natures différentes.

L'appel comme d'abus est une procédure qui existait déjà dans l'ancien droit, en France et ailleurs. Elle permettait d'accroître l'autorité de l'État sur l'Église car consistait en un mécanisme de contrôle, exercé par des juridictions étatiques sur les décisions ecclésiastiques et avant tout sur les sentences des tribunaux de l'Église. Les articles 6, 7 et 8 des Articles organiques reprirent la procédure: en cas d'abus en matière ecclésiastique, l'affaire pouvait être soumise au Conseil d'État qui déclarait s'il y avait - ou non - «abus». La Haute Assemblée prononçait une sanction morale qui, par elle-même, n'avait pas d'autre conséquence que cette «déclaration d'abus». Toutefois, l'individu ainsi sanctionné prenait conscience d'avoir encouru les foudres de l'État et, à en croire tous les témoignages de l'époque, ce verdict ne laissait pas indifférent. Au cours du XIX $x^{e}$ siècle, le Conseil fut saisi d'environ quatre cents litiges par le biais de ce recours, litiges que l'on peut répartir en quatre grandes catégories ${ }^{32}$ : les pouvoirs publics pouvaient dénoncer une prise de position peu conforme aux orientations politiques du gouvernement; les évêques furent poursuivis à plusieurs reprises pour ce motif. Un prêtre, ou un fidèle, dénonçait l'abus d'un arrêté municipal réglementant ou interdisant une manifestation extérieure du culte; le Conseil veilla à assurer l'équilibre entre liberté d'exercice public du culte et ordre public et ne permit jamais que soit prohibé un «acte essentiel de la vie religieuse», comme les convois funéraires par exemple. D'autres litiges opposaient un clerc à son évêque; le Conseil adopta systématiquement le point de vue de la hiérarchie et débouta le clerc subalterne. Enfin, des fidèles se plaignirent d'un prêtre, en cas notamment de refus de sacrement. Dès janvier 1906, le Conseil d'État estima que la procédure du recours pour abus était supprimée ${ }^{33}$, ce qui eut comme conséquence immédiate une extension nette de la liberté d'expression des ministres du culte, notamment en matière politique.

Le droit français prévoit aussi que certains agissements peuvent être passibles de poursuites pénales. Le Code pénal de 1810 consacrait une section

32. B. Basdevant-Gaudemet, Le jeu concordataire dans la France du XIXe siècle: le clergé devant le Conseil d'État, Paris, PUF, 1988.

33. Dans diverses hypothèses, les actes soumis auparavant au Conseil par la procédure du recours pour abus purent lui être désormais déférés par recours pour excès de pouvoir. Tel fut le cas des arrêtés municipaux; mais cette question de la légalité d'un arrêté municipal ne concerne qu'indirectement les éventuelles sanctions encourues par un ministre du culte. 
particulière aux «troubles apportés à l'ordre public par les Ministres des cultes dans l'exercice de leur ministère» (art. 199 à 206). Certaines dispositions sont encore en vigueur; d'autres ont disparu, ou leur formulation a été modifiée. La célébration d'un mariage religieux non précédée d'un mariage civil fut prohibée et passible de sanctions pénales dès 1792; elle le resta tout au long du XIX ${ }^{\mathrm{e}}$ siècle et l'est encore de nos jours ${ }^{34}$. Il en va de même de l'interdiction de recevoir des dons ou legs d'une personne que le ministre du culte aurait assistée en fin de vie. Dans la ligne du discours policier des Articles organiques, divers autres actes tombaient aussi sous le coup des sanctions pénales. Citons entre autres faits, les critiques proférées par un ministre du culte à l'égard de l'autorité publique, au cours d'une célébration liturgique ou dans l'exercice de ses fonctions pastorales. Le droit pénal réprimait aussi toute correspondance non autorisée avec une personne étrangère, disposition par laquelle l'autorité romaine était naturellement la première visée, etc. À cette liste non limitative s'ajoutaient naturellement d'autres chefs d'accusation, comme pour tout citoyen. Les condamnations pénales étaient souvent limitées à la somme d'un franc symbolique, peu préjudiciable au condamné; en outre, elles contribuaient à ce que le prêtre sanctionné apparaisse comme un héros auprès des fidèles.

$\mathrm{Au} \mathrm{XIX}^{\mathrm{e}}$ siècle, la procédure de mise en ouvre de ces poursuites fut discutée. Devait-on faire bénéficier le personnel religieux de la garantie prévue par l'article 75 de la Constitution de l'an VIII? Cet article avait instauré une «garantie des fonctionnaires» qui restera en vigueur jusqu'en 1870: toute poursuite à l'encontre d'un fonctionnaire pour faits relatifs à ses fonctions ne pouvait être intentée qu'après autorisation donnée par le Conseil d'État. On admit que les ecclésiastiques en bénéficiaient, même sans avoir expressément tranché la question de savoir si on leur reconnaissait (ou peut-être leur imposait) la qualité de fonctionnaires. Suite à la suppression de cette garantie, plusieurs arrêts du Conseil d'État (1880-1881) ont naturellement affirmé que les ministres du culte devaient être poursuivis selon les règles de droit commun.

La suppression de traitement pouvait constituer une troisième catégorie de sanctions. La puissance publique était-elle compétente pour suspendre le traitement d'un ministre du culte? La question fut souvent débattue, mais jamais tranchée sans ambiguité. Des suspensions furent parfois prononcées

34. V. dans ce volume la contribution de V. FORTIER, "Imam et droit pénal: de quelques infractions liées à l'exercice des fonctions cultuelles», p. 119. 
en accord avec les autorités ecclésiastiques. Dans ce cas, le prêtre ne pouvait en pratique guère s'y opposer.

Pourtant, sous le Second Empire et sous la Troisième République, il y eut des suppressions de traitement, ou des menaces de suppression. Elles furent naturellement critiquées par l'intéressé, mais l'autorité ecclésiastique dénonça aussi parfois l'attitude de l'Administration. De telles sanctions étaient infligées à un prêtre qui, dans l'exercice de sa charge pastorale, avait tenu quelques propos hostiles au gouvernement en place. Le préfet était-il alors compétent pour décider la cessation de son traitement? La question fut posée pour avis au Conseil d'État en 1883, période d'anticléricalisme virulent du gouvernement. Des prêtres avaient critiqué des «manuels d'instruction civique et morale » publiés après la loi Jules Ferry du 28 mars 1882. La consultation des procès-verbaux de la discussion au sein de la Haute Assemblée fait apparaître qu'une large majorité des conseillers estimait la suppression dénuée de base légale ${ }^{35}$. Pourtant, l'avis fut rédigé en des termes qui ne condamnaient pas la décision de suppression. Par la suite, cette sanction fut davantage une menace qu'une réalité avant qu'il ne puisse plus en être question à partir de 1905.

Le clergé catholique avait constitué le premier ordre de la Nation sous l'Ancien Régime; il conserva au XIX ${ }^{e}$ siècle une condition juridique parfois différente de celle des autres citoyens mais partageait désormais ces éléments de statut avec les autres religieux ou ministres des autres cultes reconnus. Ce statut pouvait avoir un contenu assez précis et impératif si l'on admettait que les religions constituent un «service public». Pourtant, le législateur et les tribunaux n'ont jamais ignoré les caractères spécifiques de fonctions qui ne sont pas seulement celles d'un service public de l'État. Qu'il soit autoritaire sous le Premier Empire, ou anticlérical sous la Troisième République, l'État est toujours demeuré respectueux de la liberté religieuse et de la liberté des religions, acceptant qu'un équilibre s'instaure, même si celui-ci devait parfois conduire à un relatif effacement de l'État devant les prescriptions des autorités religieuses. Le principe de liberté religieuse fut toujours strictement respecté, qu'il s'agisse de la liberté individuelle des fidèles et de leurs pasteurs ou de la liberté d'organisation interne de la confession religieuse. Quel que soit le régime juridique des relations entre l'État et les religions, le statut

35. Juridiquement, cette suppression n'était certainement pas fondée si la rémunération était la contrepartie des confiscations. D'autre part, si on admettait que la rémunération soit un traitement de fonctionnaire, ce traitement était dû «pour service fait». En l'espèce, rien n'était reproché au ministre du culte en ce qui concernait l'exercice de ses fonctions pastorales. 
des ministres du culte ne peut rester inconnu des juristes, y compris dans un régime de séparation entre l'État et les religions. Il est l'un des innombrables domaines où le dialogue entre les religions et les pouvoirs publics ne peut cesser et, comme dans tout État de droit garantissant la liberté religieuse, l'État veille à ce que les règles qu'il pose soient compatibles avec les impératifs des religions dès lors que celles-ci respectent l'ordre public. De Constantin à nos jours, en passant par la monarchie de droit divin, par le régime concordataire ou les trois départements français que constitua l'Algérie, les gouvernements se sont toujours souciés de la condition des pasteurs encadrant spirituellement les sujets ou citoyens, de leur formation, recrutement, et plus largement de leurs droits et devoirs. Cette préoccupation a constamment conduit l'État à souhaiter que les religions précisent leur propre conception de ce personnel religieux. 